\title{
Connectomics: The Dense Reconstruction of Neuronal Circuits Using Volume Electron Microscopy
}

\author{
M. Helmstaedter
}

Structure of Neocortical Circuits Group, Max Planck Institute of Neurobiology, Am Klopferspitz 18, 82152 Munich-Martinsried, Germany

The mapping of neuronal connectivity is one of the main challenges in neuroscience. Only with the knowledge of wiring diagrams is it possible to understand the computational capacities of neuronal networks, both in the sensory periphery, and especially in the mammalian cerebral cortex. Statistical circuit mapping using a combination of paired intracellular recordings and anatomical reconstructions has provided insight into the connectivity between populations of cells. To move beyond pairwise connectivity statistics, however, dense circuit mapping is required [1]. Our methods for dense circuit mapping are based on 3-dimensional electron microscopy (EM) imaging of tissue, which allows imaging nerve tissue at nanometer-scale resolution across substantial volumes (typically hundreds of micrometers per spatial dimension) using Serial Block-Face Scanning Electron Microscopy (SBEM [2,3]).

The most time-consuming aspect of circuit mapping, however, is image analysis; analysis time far exceeds the time needed to acquire the data. Therefore, we developed methods to make circuit reconstruction feasible by increasing analysis speed and accuracy, using a combination of crowd sourcing and machine learning [4]. These methods yielded a speed up in reconstruction time of 1050 fold.

We have applied these methods to circuits in the mouse retina, mapping the complete connectivity graph between almost a thousand neurons. For this, we imaged a volume sized $(120 \times 80 \times 130)$ $\mu^{3}$, spanning the ganglion cell layer, inner plexiform, inner nuclear, outer plexiform and parts of the photoreceptor layer of a piece of mouse retina at a voxel size of $(16.5 \times 16.5 \times 25) \mathrm{nm}^{3}$ using SBEM. We then reconstructed all bipolar, amacrine and ganglion cells that had their cell bodies contained in the imaged volume, amounting to a reconstruction of almost 1000 neurons or a total of approximately 0.4 meters of neuronal path length. Next, the connectivity matrix between these neurons was mapped by detecting contacts between all 500,000 cell pairs. This data provides an estimate of a large fraction of the "connectome" of the inner plexiform layer of the mouse retina.

We next improved these methods for the application to neuronal circuits in the neocortex. We acquired a first volume of neocortical data at $(12 \times 12 \times 28) \mathrm{nm}^{3}$ voxel size (Fig. 1), which was aligned to comprise a part of the neuronal circuits in the cortical input layer 4 . The dataset is currently being analyzed using online 3D EM annotation tools.

References

[1] W. Denk et al., Nat. Rev. Neuroscience. 13 (2012) 351-358.

[2] W. Denk and H. Horstmann, PLoS Biol. 2 (2004) e329

[3] K. L. Briggman et al., Nature. 471 (2011) 183-188.

[4] M. Helmstaedter et al., Nat. Neurosci. 14 (2011) 1081-1088. 


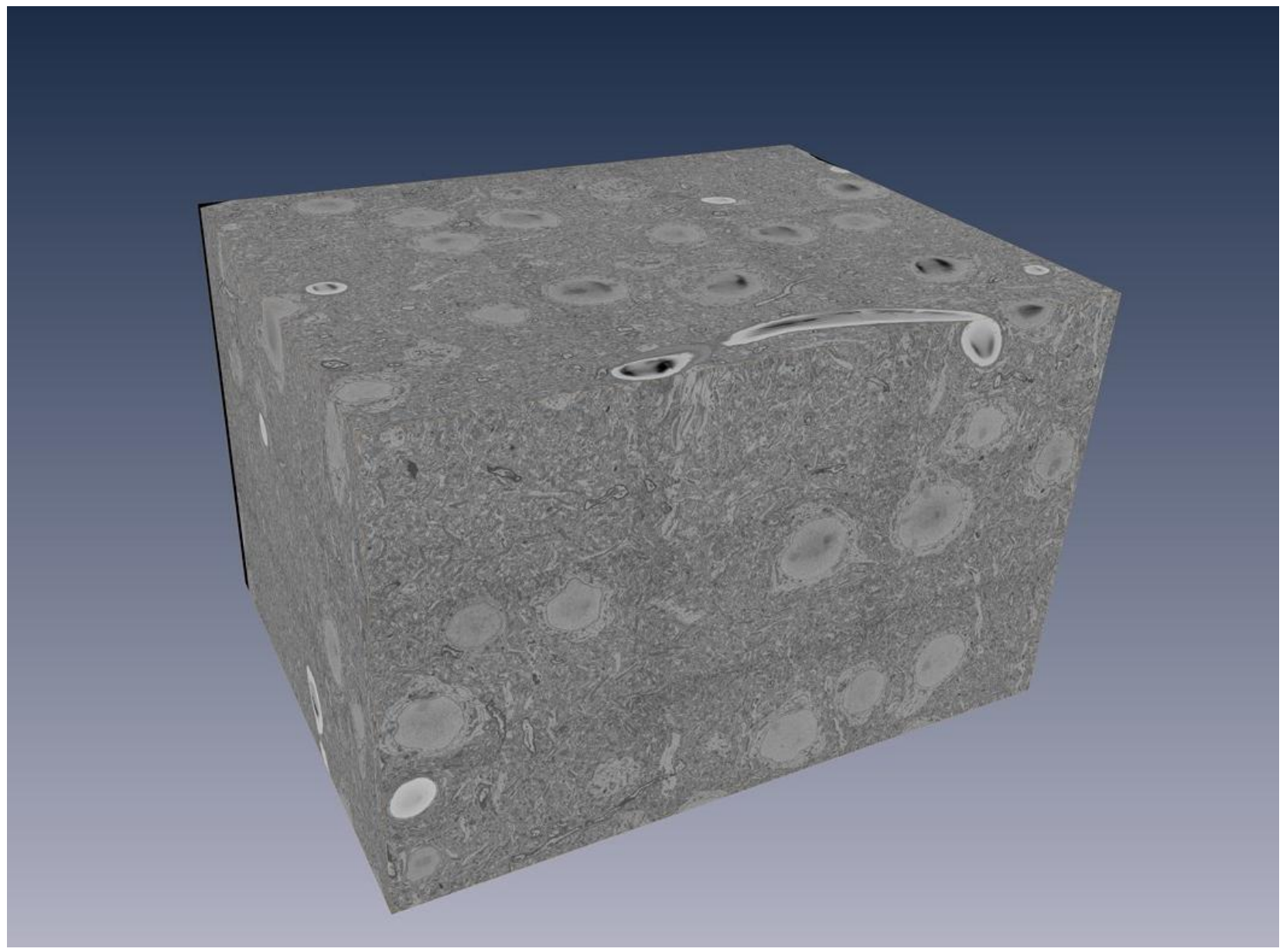

FIG. 1. Dataset from mouse primary somatosensory cortex, acquired on a Magellan 400 equipped with a custom-built Serial Blockface Electron Microscopy (SBEM) microtome and a custom amplifier for the electron detector. Voxel size is $12 \times 12 \times 28 \mathrm{~nm}^{3}$, dataset size is $100 \times 70 \times 110 \mu \mathrm{m}$ (width, height, length). Dwell time was $100 \mathrm{ns,} \mathrm{dosage} 16 \mathrm{e}-\mathrm{nm}^{2}$. Cell bodies, blood vessels, myelinated axons and apical dendrites are visible at the displayed overview resolution. 